focused on tank trucker loading operations, which were made most of the time by the truck drivers themselves, exposing the drivers to petroleum-derived fuel compounds. This kind of activity should be done by the operators of the distribution terminal, not by the truck drivers. These drivers were submitted to activities of an ambiguous nature, without qualification, adequate training, and supervision. The goal of the intervention was to change the organisation of the work in the distribution terminal as a method of reducing respiratory exposure of the truck drivers to occupational health hazards.

Methods It is a prospective descriptive case study. The period covered refers to the beginning of the inspection in the year of 2012 up to 2017. It was analysed documents that the distribution terminal has presented to labours inspection.

Result The organisation of the work at the distribution terminal changed radically. The truck drivers are not doing any operation at the terminal anymore. The distribution terminal hired new operators, modified the distribution process and yet built an occupational medical centre for their workers and for the truck drivers as a preventive measure of health.

Conclusion This case showed that is possible for Brazilians Petroleum-derived fuel distribution companies to change their work organisation and improve their environment minimising the exposure of truck drivers to occupational health hazards such as benzene.

\section{ELIMINATION, REDUCTION, AND CONTROL OF HEXAVALENT CHROMIUM RESPIRATORY EXPOSURE: A BRAZILIAN CASE}

BC Quadros*. Federal University of Rio Grande do Sul, Porto Alegre, Brazil

\subsection{6/oemed-2018-ICOHabstracts.569}

Introduction Hot dip galvanising is a method that prevents corrosion by a metallurgical bond between zinc and steel. In this industrial process, cooling and passivation baths of galvanised steel may use hexavalent chromium compounds. $\mathrm{Cr}(\mathrm{VI})$ is classified by the International Agency for Research on Cancer as Group 1 - carcinogenic to humans - and its use is restricted in most developed countries. Despite its toxicity, brazilian regulations still allow this hazardous chemical use. Thus, the development of effective control and protection rely on the understanding of occupational risks, as this paper proposes.

Method This is a descriptive quantitative cross-section case report of an industrial process: the passivation stage of galvanization. Brazilian labour inspection assigned a prohibition notice of the passivation as it was conducted, since any measures of occupational hygiene had been taken into account. Its participatory action procedure ends up preventing uncontrolled occupational exposure to $\mathrm{Cr}(\mathrm{VI})$. Secondary data source as official reports, notifications, chemical process protocols and registers from October/2016 to June/2017 have been analised. Results Before the administrative prohibition, $\mathrm{Cr}(\mathrm{VI})$ concentration in the cooling tank was 5\%; it has been eliminated. Passivation tank used to operate with $11 \%$ of $\mathrm{Cr}(\mathrm{VI})$ at $50^{\circ} \mathrm{C}$; a trial is still ongoing to adjust the concentration. The company was authorised to perform tests from 0002 to $5 \%$ at $40^{\circ} \mathrm{C}$. In this meanwhile, a satisfactory quality of galvanization was reached with a reduction of $98,84 \%$ in concentration. A ventilation system was designed to attend occupational hygiene requisites and the respiratory individual protection is effective for $\mathrm{Cr}(\mathrm{VI})$ manipulation.

Conclusion Attending the hierarchy of prevention and control measures, strategies to minimise hazards were developed, as follows: volatilisation reduction due to process, temperature and concentration modifications; ventilation system and changes in respiratory protection practices. The replacement of $\mathrm{Cr}(\mathrm{VI})$ is currently under study.

\section{PHYSIOLOGICAL EFFECTS OF WEARING POWERED AIR- PURIFYING RESPIRATORS WITH DIFFERENT BLOWER SYSTEM}

${ }^{1}$ Shoko Kawanami*, ${ }^{2}$ Yuichiro Tanaka, ${ }^{2}$ Shota Tabuchi, ${ }^{2}$ Jinro Inoue, ${ }^{3}$ Hiroyuki Hayashi, ${ }^{3}$ Saki Hanaoka, ${ }^{3}$ Norikazu Kurano, ${ }^{2}$ Seichi Horie. 'Occupational Health Training Centre, University of Occupational and Environmental Health, Japan; ${ }^{2}$ Department of Health Policy and Management, University of Occupational and Environmental Health, Japan; ${ }^{3}$ Shigematsu Works Co., Ltd

\subsection{6/oemed-2018-ICOHabstracts.570}

Introduction Inhalation resistance of respirators may induce additional burden for workers breathing. Powered air-purifying respirators (PAPR) are considered to reduce this burden by assisting inhalation with powered fan. While there are mainly two types of blower system; breath response type and constant air flow type, no study has been conducted to clarify the difference of their physiological effects as far as we searched. We conducted this study to elucidate the physiological effects of wearing different types of PAPR by measuring physiological parameters and the microclimate inside the PAPRs.

Methods Eight healthy males participated in the study. They took a 40 minutes-exercise with ergometer at 20, 30, 40\% and $50 \% \mathrm{VO}_{2} \max$ while wearing three types of masks;

- half mask facepiece with breath response type (BR-PAPR),

- half mask facepiece with constant air flow type (CF-PAPR) and

- mask of expiration gas analyzer (control).

We continuously measured their auditory canal temperature $\left(t_{\mathrm{ac}}\right)$, heart rate (HR) and $\mathrm{SpO}_{2}$. We also measured the microclimates (temperature, humidity, $\mathrm{O}_{2}, \mathrm{CO}_{2}$ ) inside the two PAPRs. Their subjective symptoms were also surveyed. Statistical analysis was performed by IBM SPSS Statistics22.0.

Result There were few differences in the trend of $t_{\mathrm{ac}}$, HR and $\mathrm{SpO}_{2}$ among the masks. $\mathrm{SpO}_{2}$ gradually declined but remained within normal level. The temperature and humidity inside masks were higher in BR-PAPR. During inhalation period, the proportion of time which $\mathrm{O}_{2}$ concentration became lower than $18 \%$ or which $\mathrm{CO}_{2}$ concentration exceeds $3 \%$ inside the respirators were lower in CF-PAPR. The participants' perception of hotness or humidity coincided with the microclimates. Discussion BR-PAPR is known for the better durability of battery compared with CF-PAPR. There were few differences in physiological parameters among those respirators. However, with respect to the microclimate inside respirators, CF-PAPR was better than the BR-PAPR, hence it may affect comfort of the workers. 